ARTICLE

\title{
Spatial Distribution Measurement of Neutrons Produced by 120-GeV Proton Beam in Concrete Shield
}

\author{
Hiroshi YASHIMA ${ }^{1 *}$, Yoshimi KASUGAI ${ }^{2}$, Norihiro MATSUDA ${ }^{2}$, Hiroshi MATSUMURA ${ }^{3}$, Shun SEKIMOTO ${ }^{1}$, Akihiro \\ TOYODA ${ }^{3}$, Hiroshi IWASE ${ }^{3}$, Nikolai MOKHOV ${ }^{4}$, Anthony LEVELING ${ }^{4}$, David BOEHNLEIN ${ }^{4}$, Kamran VAZIRI $^{4}$ \\ Koji $\mathrm{OISHI}^{5}$, Hiroshi NAKASHIMA ${ }^{2}$ and Yukio SAKAMOTO ${ }^{2}$
}

\author{
${ }^{1}$ Kyoto University Research Reactor Institute, Kumatori-cho Sennan-gun Osaka 590-0494, Japan \\ ${ }^{2} J a p a n$ Atomic Energy Agency, 2-4 Shirakata Shirane, Tokai-mura, Ibaraki 319-1195 Japan \\ ${ }^{3}$ High Energy Accelerator Research Organization (KEK), 1-1 Oho, Tsukuba, Ibaraki 305-0801 Japan \\ ${ }^{4}$ Fermi National Accelerator Laboratory, Fermilab, Batavia, IL 60510-5011, USA \\ ${ }^{5}$ Shimizu Corporation, 4-17, Etchujima 3-chome, Koto-ku, Tokyo 135-8530, Japan
}

\begin{abstract}
The shielding experiment was performed at the anti-proton (pbar) production target station in Fermi National Accelerator Laboratory to obtain benchmark data for various transport calculation codes. At the pbar target station, an antiproton production target, consisted of Inconel and copper disks, were irradiated by $120 \mathrm{GeV}$ proton beam. The beam intensity was $2.3 \times 10^{12}$ proton per second. The iron and concrete shields were placed on the upper side of the target. The thickness of iron and concrete shields were 188 and $122 \mathrm{~cm}$, respectively. Aluminum, bismuth, and indium samples were placed in the concrete shields to measure the neutron energy spectra in the concrete shields. After irradiation, induced activities in samples were measured by using HPGe detector. The reaction rate spatial distributions of ${ }^{27} \mathrm{Al}(\mathrm{n}, \alpha){ }^{24} \mathrm{Na},{ }^{209} \mathrm{Bi}(\mathrm{n}, \mathrm{xn}){ }^{203,204,205,206} \mathrm{Bi}$, and ${ }^{115} \mathrm{In}\left(\mathrm{n}, \mathrm{n}^{\prime}\right){ }^{115 \mathrm{~m}} \mathrm{In}$ reactions were measured in the concrete shields. The neutron energy spectra in the concrete shields were also obtained by unfolding the measured reaction rates with the aid of neutron induced reaction cross sections.
\end{abstract}

\section{KEYWORDS: Activation Detector, Fermi National Accelerator Laboratory, Shieding experiment}

\section{Introduction}

The Japanese and American Study of Muon Interaction and Neutron detection (JASMIN) collaboration has been organized to study radiation effects associated with the high energy particle beams at the Fermi National Accelerator Laboratory (FNAL) $)^{1)}$. The collaboration aims to study the behavior of secondary particles generated from beam losses in high-energy accelerators: particle fluxes and spectra, activities in air, water and materials, and radiation damage of components. Although considerable work has been done by similar experiments in the past ${ }^{2-5}$, only a few have attempted such measurements with beam energies exceeding $1 \mathrm{GeV}^{6,7)}$. In this energy region, most simulation codes implement a transition of reaction models from intermediate to high energies $^{8-11)}$. The collection of reaction data and comparison of the measured data to simulations is the basic methodology for benchmarking studies to improve the reaction models.

The data discussed here were taken at the anti-proton (Pbar) production hall at FNAL. The Pbar target produces anti-protons for FNAL experiments in the Tevatron collider using a $65-\mathrm{kW}$ proton beam of momentum $120 \mathrm{GeV} / \mathrm{c}$. The spatial distribution of nuclear interactions and the neutron flux behind the shielding have been measured at this facility and an analysis comparing them with Monte Carlo

*Corresponding Author, E-mail: yashima@rri.kyoto-u.ac.jp (C2012 Atomic Energy Society of Japan, All Rights Reserved calculations has been performed. Preliminary results are reported ${ }^{12-15)}$ and the studies are ongoing.

In this study, the spatial distributions of reaction rates of samples which were placed in the concrete shield were measured to obtain the experimental data for high energy secondary particle transport from the target bombarded by high energy protons. The neutron energy spectra in the concrete shield were also obtained by unfolding the measured reaction rates with the aid of neutron induced reaction cross sections.

\section{Experiment}

Figure 1 shows the cross sectional view of the Pbar target station. At this station, an antiproton production target made of inconel and copper disks was irradiated by $120 \mathrm{GeV}$ protons. Average beam intensity was $2.3 \times 10^{12}$ protons $/ \mathrm{sec}$. In downstream of the target, a correction lens, collimator and a pulsed magnet are placed to focus, collimate, and extract the produced antiprotons. The remaining protons and secondary particles are absorbed by the dump placed in downstream of the pulsed magnet. Iron and concrete shields are placed upper side of the target and magnets. The thicknesses of iron and concrete shields above the target are 188 and $122 \mathrm{~cm}$, respectively. The concrete shields were renewed to install the activation samples. Air gap of $179 \mathrm{~cm}$ height is between the iron and concrete shields. The distance 
between the target and the lower surface of iron shields is 46 $\mathrm{cm}$. The activation samples (Aluminum, bismuth, and indium) were installed in concrete shields to measure spatial distribution of secondary neutrons. Figure 2 shows the position of installed samples in concrete shields. There were 4 holes which were placed just above the target for hole B and in 136-cm upstream and 136, 204-cm downstream from the target for hole A, C, D, respectively. Seven samples were installed into each hole. The physical properties of radioactive nuclides used in this study are listed in Table $\mathbf{1}^{16)}$.

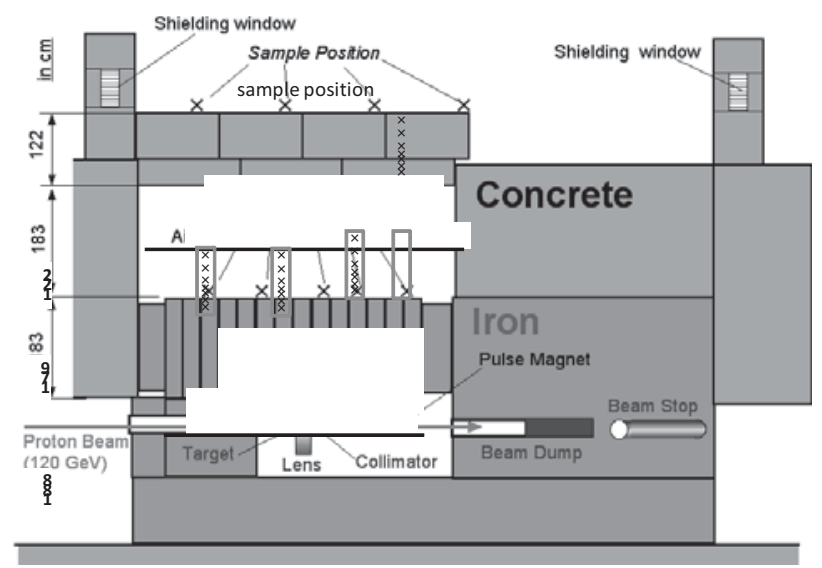

Fig. 1 The cross sectional view of the Pbar target station.

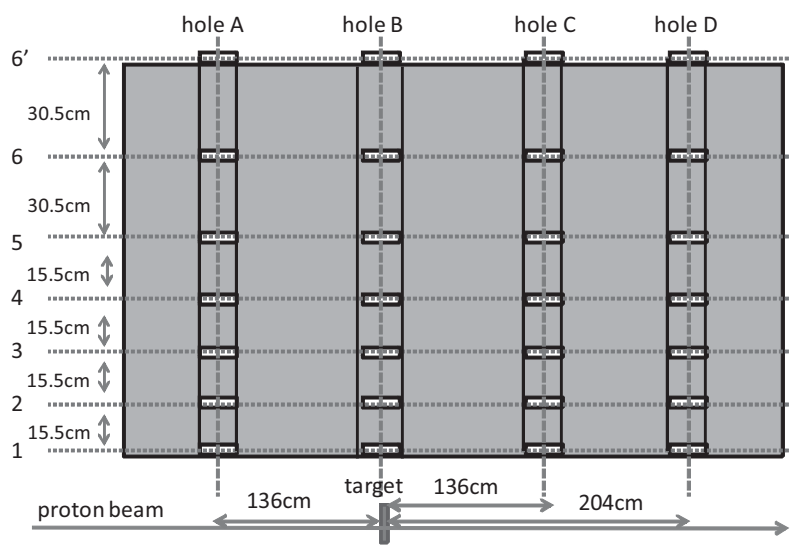

Fig. 2 The position of installed samples in concrete shields.

Table 1 The physical properties of radioactive nuclides.

\begin{tabular}{cccc}
\hline Reaction & Half-life & $\begin{array}{c}\text { Gamma-ray } \\
\text { Energy[keV] }\end{array}$ & $\begin{array}{c}\text { Branching } \\
\text { Ratio[\%] }\end{array}$ \\
\hline${ }^{27} \mathrm{Al}(\mathrm{n}, \alpha)^{24} \mathrm{Na}$ & $14.959 \mathrm{~h}$ & 1368.63 & 100 \\
\hline${ }^{209} \mathrm{Bi}(\mathrm{n}, 4 \mathrm{n}){ }^{206} \mathrm{Bi}$ & $6.243 \mathrm{~d}$ & 803.1 & 98.9 \\
\hline${ }^{209} \mathrm{Bi}(\mathrm{n}, 5 \mathrm{n}){ }^{205} \mathrm{Bi}$ & $15.31 \mathrm{~d}$ & 703.45 & 31.1 \\
\hline${ }^{209} \mathrm{Bi}(\mathrm{n}, 6 \mathrm{n}){ }^{204} \mathrm{Bi}$ & $11.22 \mathrm{~h}$ & 374.76 & 81.8 \\
\hline${ }^{209} \mathrm{Bi}(\mathrm{n}, 7 \mathrm{n})^{203} \mathrm{Bi}$ & $11.76 \mathrm{~h}$ & 820.23 & 29.6 \\
\hline${ }^{115} \mathrm{In}\left(\mathrm{n}, \mathrm{n}^{\prime}\right)^{115 \mathrm{~m}} \mathrm{In}$ & $4.486 \mathrm{~h}$ & 336.241 & 45.9 \\
\hline
\end{tabular}

\section{Analysis}

After irradiation, the samples were taken to a counting laboratory equipped with Canberra high-purity germanium (HPGe) detectors. The HPGe detectors are used to characterize the gamma-ray spectra and measure the decay curves. In this way, the nuclides induced in the foils are identified. Reaction rates for each nuclide are determined after being corrected for the peak efficiency of the HPGe detectors and the beam current fluctuation during the irradiation. One of the HPGe detectors was calibrated by Canberra $^{17)}$. The peak efficiency of the calibrated HPGe detector was determined using Canberra's LabSOCS software $^{18)}$. For the non-calibrated detectors, the peak efficiency was determined from the ratios of their peak counting rates to that of the calibrated detector. The components of estimated errors in reaction rates were the counting statistics and detector efficiency.

To compare experimental results with simulation, neutron energy spectra on sample position were calculated by the

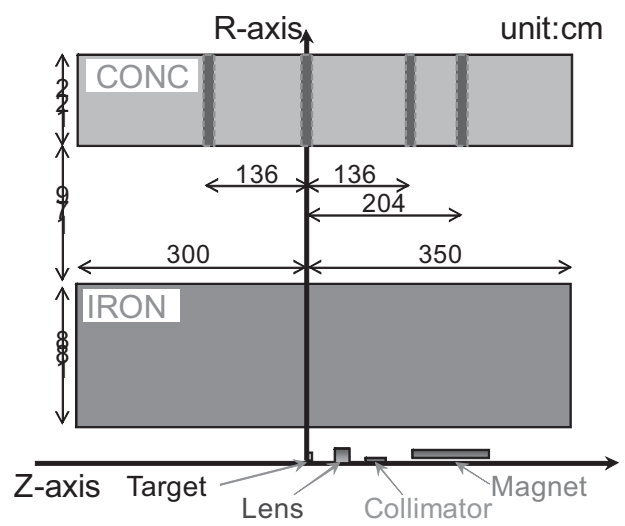

Fig. 3 Two-dimensional(R-Z) simplified geometry.

PHITS code ${ }^{10,11)}$. In this PHITS calculation, the simplified two-dimensional(R-Z) geometry was adopted as shown in Figure 3. For comparison, the calculated neutron energy spectra were converted to reaction rates by multiplying them with reaction cross sections ${ }^{19,20)}$ as shown in Figure 4.

The neutron energy spectra were also obtained by unfolding the measured reaction rates with the aid of response function. The SAND II $\operatorname{code}^{21)}$ was used for this unfolding. The initial guess spectra for this unfolding were obtained from the PHITS calculation described above. The reaction cross section data shown in Figure $\mathbf{4}$ were adopted as the response functions.

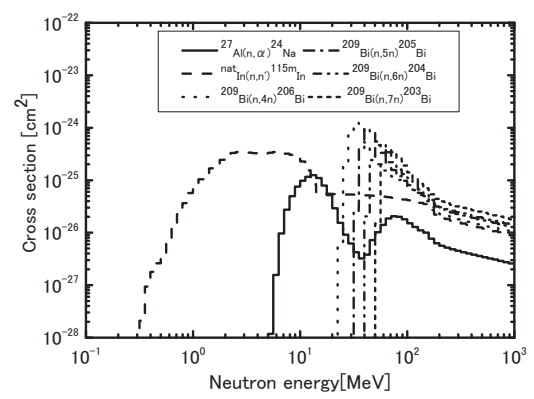

Fig. 4 Reaction cross sections of produced nuclides. 


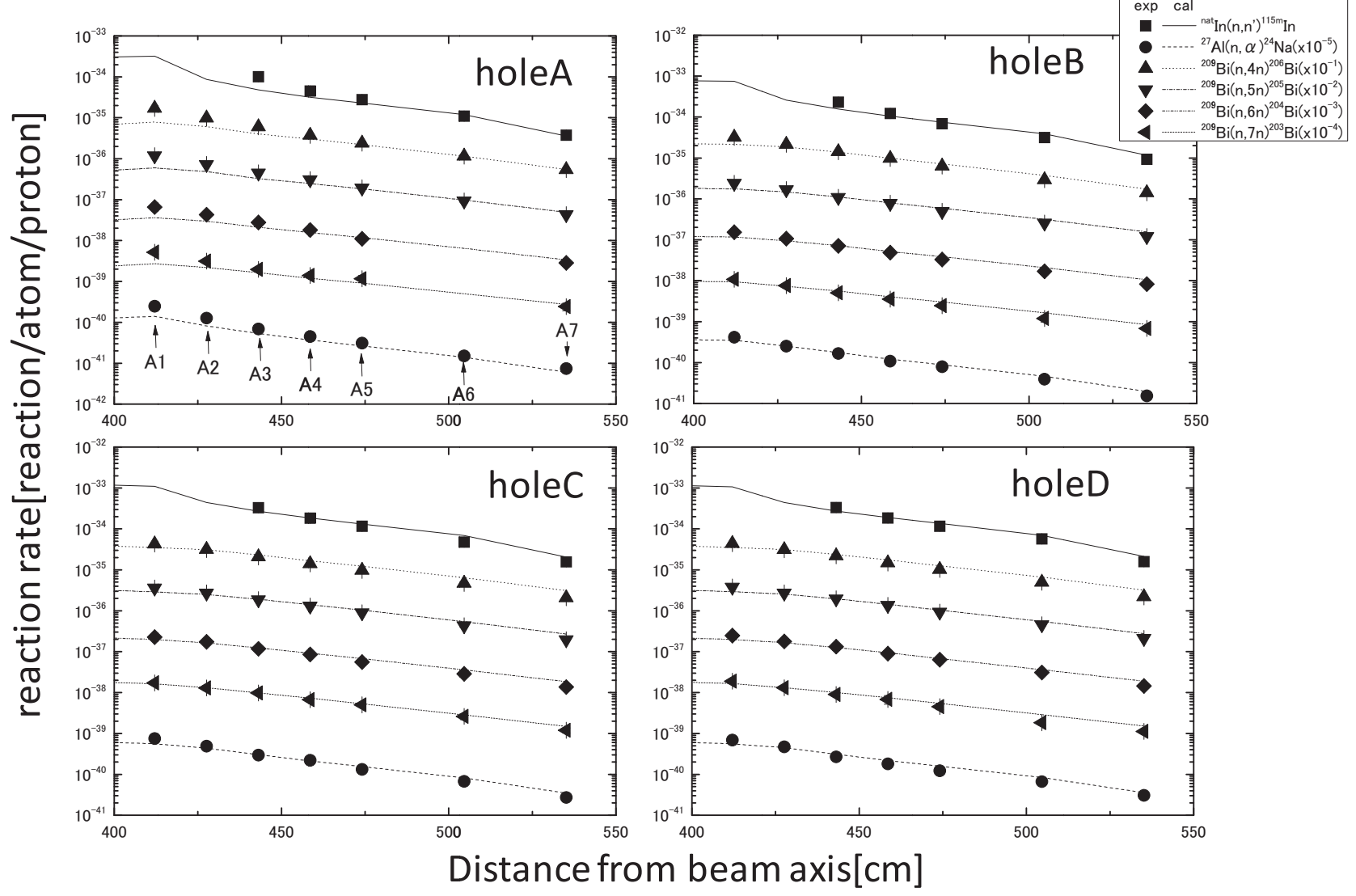

Fig. 5 The spatial distribution of neutron-induced reaction in concrete shield.

\section{Results and Discussion}

\section{Spatial distribution of neutron-induced reaction in} concrete shield

The spatial distributions of neutron-induced reactions in concrete shield are shown in Figure 5. The points indicate experimental results. The lines indicate calculation results. In Figure 5, the calculation results generally agree with

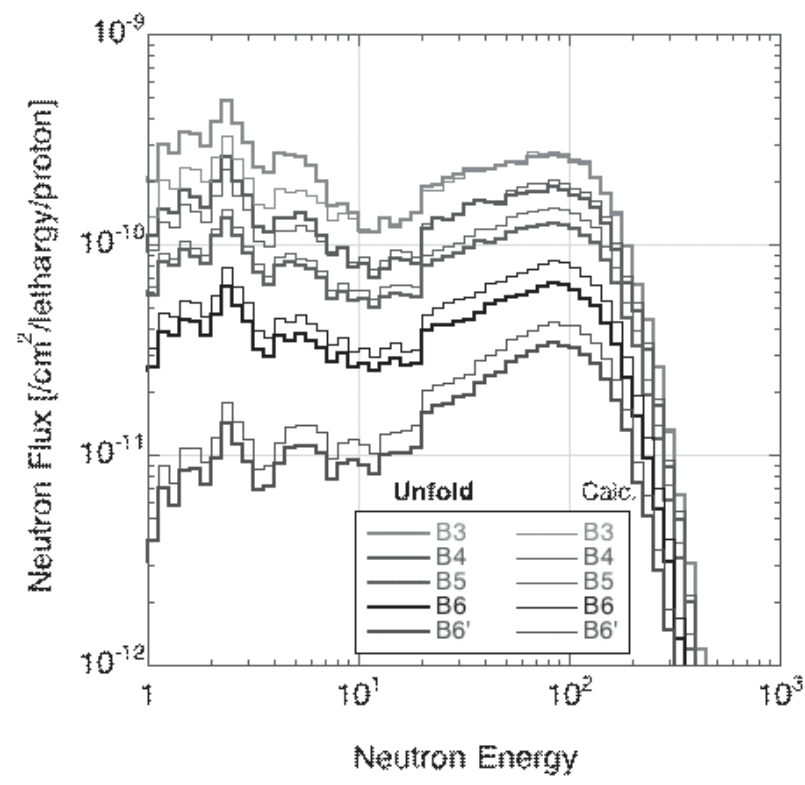

Fig. 6 The neutron energy spectra in concrete shield (hole B). experimental results, but there are some difference between calculation results and experimental results. Further

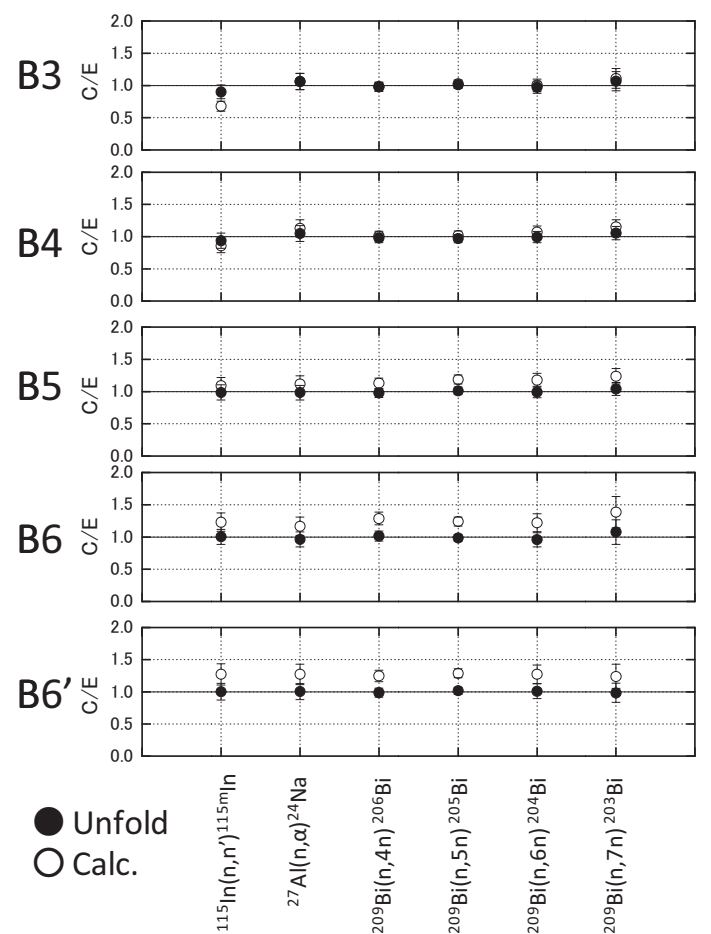

Fig. 7 The $\mathrm{C} / \mathrm{E}$ values for the reaction rates by unfolded spectra and that by calculated spectra. 
calculation analyses are required for investigation of behavior of secondary neutron in concrete shield, because the simplified two-dimensional(R-Z) geometry was adopted in this calculation.

\section{Neutron energy spectra in concrete shield}

The neutron energy spectra in concrete shield are shown in Figure 6. The bold lines indicate unfolded energy spectra. The narrow lines indicate calculated energy spectra.

The $\mathrm{C} / \mathrm{E}$ values of the reaction rates obtained form unfolded spectra in closed black circles and those from calculated spectra in open circles are shown in Figure 7. In Figure 7, the reaction rates by unfolded spectra agree with measured reaction rates for all reactions.

\section{Conclusions}

The spatial distributions of reaction rate of samples in concrete shield were measured. The neutron energy spectra in the concrete shields were also obtained by unfolding the measured reaction rates with the aid of neutron induced reaction cross sections.

These experimental results will be useful as benchmark data to investigate the accuracy of various transport calculation codes.

\section{Acknowledgment}

This work was supported by a grant-in-aid from the Ministry of Education, Science and Culture (KAKENHI 19360432) in Japan. Fermilab is a US Department of Energy Laboratory operated under Contract DE-AC02-07CH11359 by the Fermi Research Alliance, LLC.

\section{References}

1) H. Nakashima, et al., "Experimental studies of shielding and irradiation effects at high-energy accelerator facilities" Nucl.Technol., 168[2], 482(2009).

2) N. Nakao, et al., "Transmission through shields of quasi-monoenergetic neutron generated by $43-$ and 68 $\mathrm{MeV}$ protons-I: Concrete shielding experiment and calculation for practical application", Nucl. Sci. Eng., 124, 228(1996).

3) H. Nakashima, et al., "Transmission through shields of quasi-monoenergetic neutron generated by $43-$ and $68-\mathrm{MeV}$ protons-II: Iron shielding experiment and analysis for investigating calculational method and cross-section data", Nucl. Sci. Eng., 124, 243(1996).

4) T. Nunomiya, et al., "Measurement of deep penetration of neutrons produced by $800-\mathrm{MeV}$ proton beam through concrete and iron at ISIS", Nucl. Instrum. Meth., B179, 89(2001).

5) N. Nakao, et al., "Arrangement of high-energy neutron irradiation field and shielding experiment using $4 \mathrm{~m}$ concrete at KENS", Radiat. Prot. Dosim., 116, 553(2005).
6) H.Nakashima, et al., "Current status on AGS spallation target experiment" Proc. of the Shielding Aspects on Accelerator, Target and Irradiation Facilities -SATIF6, SLAC, Apr. 10-12, 2002.

7) N. Nakao, et al., "Measurement of neutron energy spectra behind shielding of a $120 \mathrm{GeV} / \mathrm{c}$ hadron beam facility" Nucl. Instrum. Meth., A562, 950(2006).

8) N. V. Mokhov, "The Mars code system user's guide", FERMILAB-FN-628 (1995),

9) N.V. Mokhov, S.I. Striganov, "The MARS overview", Fermilab-Conf-07-008-AD (2007).

10) H. Iwase et al., "Development of General-Purpose Particle and Heavy Ion Transport Monte Carlo Code" J. Nucl. Sci. Technol. 39, 1142(2002).

11) K. Niita, et al., "PHITS-a particle and heavy ion transport code system" Radiat. Meas., 41, 1080(2006).

12) T. Sanami et al., "Shielding Experiments at High Energy Accelerators of Fermilab (I) - Dose rate around high intensity muon beam - “, Prog. Nucl. Sci. Technol., Vol.1, 44 (2011).

13) H. Yashima et al., "Shielding Experiments at High Energy Accelerators of Fermilab (II) - Spatial distribution measurement of reaction rate behind the shield and its application for Moyer model - ", Prog. Nucl. Sci. Technol., Vol.1, 48 (2011).

14) M. Hagiwara et al., "Shielding Experiments at High Energy Accelerators of Fermilab(III) - Neutron spectrum measurements in intense pulsed neutron fields of the $120-\mathrm{GeV}$ proton facility using a current Bonner sphere technique - ", Prog. Nucl. Sci. Technol., Vol.1, 52 (2011).

15) N. Matsuda et al., "Shielding Experiments at High Energy Accelerators of Fermilab (IV) - Calculation analysis - ", Prog. Nucl. Sci. Technol., Vol.1, 57 (2011).

16) R. B. Firestone, Table of Isotopes, 8th ed., John Wiley and Sons, Inc., New York 1996.

17) R. Venkataraman, F. Bronson, V. Atrashkevich, M. Field, B. M. Young, "Improved detector response characterization method in ISOCS and LabSOCS", $J$. Radioanal. Nucl. Chem., 264, 213 (2005).

18) F. L. Bronson, "Validation of the accuracy of the LabSOCS software for mathematical efficiency calibration of Ge detectors for typical laboratory samples", J. Radioanal. Nucl. Chem., 255, 137 (2003).

19) F. Maekawa et al., "Production of a Dosimetry Cross Section Set Up to $50 \mathrm{MeV}$ ", Proc. 10th Int. Symp. Reactor Dosimetry, September 12-17,1999, Osaka, Japan, p.417, American Society for Testing and Materials (2001).

20) F. Maekawa et al., "Analysis of a Neutronic Experiment on a Simulated Mercury Spallation Neutron Target Assembly Bombarded by Giga-Electron-Volt Protons", Nucl. Sci. Eng.,150,99(2005).

21) W. N. McElroy, S. Berg, T. Crockett and R. G. Hawkins, "A computer-automated Iterative Method for Neutron Flux Spectra Determination by Foil Activation", Air Force Weapons Laboratory, (1967) 